Article

\title{
Improving Spinnability of Hyper-Coal Derived Spinnable Pitch through the Hydrogenation with 1,2,3,4-Tetrahydroquinoline
}

\author{
Jianxiao Yang ${ }^{1,2, *}(\mathbb{D})$, Wei $\mathrm{Wu}^{1}$, Xiaxiang Zhang ${ }^{1}$, Kui Shi ${ }^{1}$, Xuanke $\mathrm{Li}^{1,2}$ and Seong-Ho Yoon ${ }^{3}$ \\ 1 College of Materials Science and Engineering, Hunan University, Changsha 410082, China; \\ weiwu@hnu.edu.cn (W.W.); zhangxiax@hnu.edu.cn (X.Z.); skhnu123@163.com (K.S.); \\ xuankeli@hnu.edu.cn (X.L.) \\ 2 Hunan Province Key Laboratory for Advanced Carbon Materials and Applied Technology, \\ Hunan University, Changsha 410082, China \\ 3 Institute for Materials Chemistry and Engineering, Kyushu University, 6-1 Kasugakoen, Kasuga 8168580, \\ Fukuoka, Japan; yoon@cm.kyushu-u.ac.jp \\ * Correspondence: yangjianxiao@hnu.edu.cn; Tel.: +86-152-1100-5929
}

Received: 20 July 2018; Accepted: 16 August 2018; Published: 19 August 2018

\begin{abstract}
The proper hydrogenation of Hyper-coal (HPC) using 1,2,3,4-tetrahydroquinoline (THQ) was able to decrease the oxygen content and adjust the molecular structure of HPC for preparing the spinnable pitch with high softening point (SP). The spinnable pitch prepared from the THQ-soluble (QS) fraction of HPC as a precursor consisted of more naphthenic carbon groups than that prepared from the 1-methylnaphthalene (1-MN) soluble (MNS) fraction of HPC. The HPC-QS derived spinnable pitch showed excellent spinning performance even though the SP of $260^{\circ} \mathrm{C}$, and the tensile strength of the resultant carbon fiber was increased to $1350 \mathrm{MPa}$ with a diameter around $8 \mu \mathrm{m}$ under the only carbonization temperature of at $800{ }^{\circ} \mathrm{C}$ for $5 \mathrm{~min}$.
\end{abstract}

Keywords: hyper-coal; hydrogenation; spinnable pitch; carbon fiber

\section{Introduction}

A steady and sharp increase in the application market and prospect of carbon fibers ( $\mathrm{CFs})$ in the future can be predicted because of their low density and excellent mechanical properties, good electrical and thermal properties. However, the prospects and large-scale consumption of CFs are still limited by their high cost and insufficient properties, which come from the expensive raw material, low precursor yield and complex production processes of CFs. Hence, there is much room to reduce the production cost and improve the property of CFs, and it is great challenge to expand a huge market for CFs as a low-carbon green material in environmental and low-energy applications. Recently, many researchers concentrate on developing CFs and paid more attention to develop possible routes towards the production of suitable low-cost CFs with a medium tensile strength of $1700 \mathrm{MPa}$ for large-scale automotive use, which necessitates a large reduction in cost of industrial grade CFs from about $\$ 30 / \mathrm{kg}$ to the acceptable and feasible target range of $\$ 11-15 / \mathrm{kg}$. Currently, there are mesophase pitch-based and polyacrylonitrile (PAN)-based CFs that can be used in the automotive body in terms of mechanical properties. However, the commercialization is limited by their high cost. Actually, the cost of precursor generally accounts for almost $50 \%$ of the total production cost of CFs [1], which implies that developing a low-price precursor may be the most possible and effective method to reduce these high manufacturing costs. Thus, the feasible route for achieving the target of low-cost CFs production is to prepare the fiber precursors using cheap raw materials. 
Currently, there are a number of previous researchers attempting to develop the novel low-cost CFs using lignin [1], liquefied wood [2], anthracene oil [3], biomass tar [4] and pyrolysis fuel oil (PFO) [5] and ethylene bottom oil (EO) [6,7] as inexpensive raw materials. Among them, only a few researches have successfully obtained their CFs with satisfactory mechanical properties. In particular, our group reported the development of a novel isotropic spinnable pitch composed of linear molecules synthesized through bromination-dehydrobromination of $\mathrm{EO}$ and coal tar pitch. The properties of the resultant $\mathrm{CF}$ s were suitable for their application in vehicles $[6,7]$. However, the bromination-dehydrobromination reaction process requires special handling conditions and produces strong acidic byproducts due to the large amounts of potentially dangerous bromine reagents. Herein, much of the current work focus on developing a fast, cheap and simple method for the fabrication of pitch based CFs using one alternative and low-price raw material.

Recently, we tried to use Hyper-coal (HPC) as a low-price and effective raw material for the development of pitch based CFs due to the HPC is free of minerals and exhibits highly fluid $[8,9]$. There should be various forms of coal-derived byproducts. For example, coal tar and coal tar pitch are most typical coal derived byproducts which have been intensively and thoroughly studied to utilize them for the production of various functional carbon materials such as carbon fiber, graphite electrodes and activated carbon [10-12]. Coal tar is a typical byproduct of coal carbonization which can be produced through the coal pyrolysis over $800{ }^{\circ} \mathrm{C}$ in coke oven [13], while HPC is a very special coal liquefied products which has been only experienced the heat treatment of less than $400{ }^{\circ} \mathrm{C}$ [14]. From these reasons, HPC has very different characteristics from coal tar. Coal tar, which is coal pyrolyzed byproduct at over $800{ }^{\circ} \mathrm{C}$, usually has characteristics such as molecular compositions of relatively low heterogeneous atoms, high aromaticity, 3-4 condensed aromatic ring compounds, relatively small molecular weight distribution, almost no double bond compounds, and almost no 5-membered aromatic ring compounds [15]. However, HPC, which is a coal extracted one at less than $400^{\circ} \mathrm{C}$, usually has characteristics of molecular compositions of relatively high heterogeneous atoms $(\mathrm{O}, \mathrm{N})$, high aliphatic contents, broad compositions of 1-8 condensed aromatic ring compounds, relatively large molecular weight distribution, some amounts of double or triple bond compounds, and some amounts of 5 membered aromatic ring compounds [16]. From such points, HPC has much more difficult problems to prepare the spinnable pitch precursor with high softening point compared to coal tar. Therefore, in our previous work, some approaches have been attempted to prepare the HPC-derived spinnable pitch. The previous results suggested that the HPC can obtain most suitable spinnable pitch precursor of the appropriate molecular weight for $\mathrm{CFs}$ production through the proper solvent fractionation method. In addition, the 1-methylnaphthalene (1-MN) soluble (MNS) fraction of HPC was successfully produced the pitch based CFs as an inexpensive and effective raw material [9]. However, the softening point (SP) of MNS-derived pitch was limited below $210{ }^{\circ} \mathrm{C}$ due to the high oxygen content and molecular structure. In order to improve the spinnability of pitch and the tensile strength of their carbon fiber, we figure out that the proper hydrogenation of HPC using 1,2,3,4-tetrahydroquinoline (THQ) solvent can be expect to decrease the oxygen content and adjust the molecular structure of HPC for preparing the spinnable pitch with higher SP. Therefore, in this work, we describe the preparation and characterization of pitch-based CFs using the QS fraction of HPC as a precursor, and present a comparison of the pitches and CFs prepared from MNS and QS fractions of HPC.

\section{Materials and Methods}

\subsection{Materials}

HPC was supplied by Kobe Steel Co. Ltd. (Kobe, Japan), as a raw material, which was produced from the Australian bituminous coal (hard-coking coal). The QS fraction of HPC was prepared by hydrogenation of THQ/HPC with weight ratio of 5 at $450{ }^{\circ} \mathrm{C}$ for $20 \mathrm{~min}$ in the stainless steel autoclave reactor with a $0.1 \mathrm{MPa}$ nitrogen pressure. The mixtures were then filtered with $1 \mu \mathrm{m}$ 
polytetrafluoroethylene filter paper at room temperature and the soluble fraction was evaporated at $200{ }^{\circ} \mathrm{C}$ with a vacuum degree of $133 \mathrm{~Pa}$ for recycling the THQ solvent. In order to compare with the properties of MNS-derived pitch and carbon fiber, the MNS fraction of HPC was also prepared by 1-MN solvent extraction at $70^{\circ} \mathrm{C}$ for $1 \mathrm{~h}$ as reported in previous paper [9]. The resulting two precursors were designated to HPC-MNS and HPC-QS respectively.

\subsection{Preparation of Pitches and Carbon Fibers}

Although the MNS or QS fractions of HPC with low SP might be used as a precursor for spinning into pitch fibers, the volatile matters of MNS or QS fractions of HPC are detrimental to their spinnability and the obtained pitch fibers are easy to be fused together after the stabilization and carbonization treatments. Meanwhile, the resultant CFs scarcely have mechanical properties. A suitable spinnable pitch precursor should have a high SP for easy stabilization of pitch fibers as well as adequate spinnability [17]. Therefore, the MNS and QS fractions from HPC were continuously heat treated by thin layer evaporation with $500 \mathrm{~Pa}$ vacuum degree at different temperatures below $300{ }^{\circ} \mathrm{C}$ for different soaking times to remove their volatile matters and adjust their SPs of 210,240 , and $260^{\circ} \mathrm{C}$. Then, the prepared pitches were spun into pitch fibers by a melt spinning method with the spinning temperature of their $\mathrm{SP}+50^{\circ} \mathrm{C}$. The diameter and length of spinneret is $0.2 \mathrm{~mm}$ and $0.4 \mathrm{~mm}$, respectively. Finally, the spun pitch fibers were stabilized in a $200 \mathrm{~mL} / \mathrm{min}$ air-flow rate from room temperature to $270{ }^{\circ} \mathrm{C}$ for $1 \mathrm{~h}$ with a heating rate of $0.5^{\circ} \mathrm{C} / \mathrm{min}$. Additionally, the stabilized fibers (SFs) were successively carbonized in a $100 \mathrm{~mL} / \mathrm{min}$ nitrogen flow rate from room temperature to $800{ }^{\circ} \mathrm{C}$ for 5 min with a heating rate of $5{ }^{\circ} \mathrm{C} / \mathrm{min}$.

\subsection{Characterization of Pitches and Carbon Fibers}

A brief description of characterizations of the obtained pitches and CFs is given here, while detailed descriptions are given elsewhere [9]. The SP of raw materials and pitches were measured by thermal mechanical analyzer (TMA, Exstar SII, TMA/SS6300, Nanotechnology, Inc., Chiba, Japan). The physical and chemical properties of precursors and prepared pitches were characterized using solid ${ }^{13} \mathrm{C}$ nuclear magnetic resonance $\left({ }^{13} \mathrm{C}-\mathrm{NMR}\right.$, ECA400, JEOL, Tokyo, Japan), time of flight mass spectrometry (TOF-MS, JMS-S3000, JOEL, Tokyo, Japan), X-ray diffraction analyzer (XRD, RINT Ultimate-III, Rigaku, Tokyo, Japan), and thermogravimetry analyzer (TGA, Exstar SII, TG/DTA6300, Nanotechnology, Inc., Chiba, Japan). The oxygen contents of samples were measured by the equation of $\mathrm{O}=100-\mathrm{C}-\mathrm{H}-\mathrm{N}$, the carbon aromaticity degree $\left(f_{a}\right)$ of samples were calculated from ${ }^{13} \mathrm{C}$-NMR result and the average molecular weight $\left(M_{w}\right)$ of samples was evaluated from TOF-MS result. The diameters of the obtained CFs were determined using a laser analyzer (M550A, Anritsu, Japan), and the tensile strengths of the obtained CFs were measured according to JIS7601 standards (single-fiber method) via a strength tester apparatus (Tensilon/UTM-II-20, TOYO BOLDWIN Co., Ltd., Tokyo, Japan). The morphologies of the obtained CFs were observed by scanning electron microscopy (SEM, JSM-6700F, JOEL, Tokyo, Japan).

\section{Results and Discussion}

\subsection{Analysis of HPC-Derived Precursors}

The general characteristics of HPC, HPC-MNS and HPC-QS are summarized in Table 1. Following solvent extraction or hydrogenation of HPC, the SPs of the MNS and QS fractions decreased. In addition, the SP of HPC-QS was lower than that of HPC-MNS, while the yield of HPC-QS was slightly higher than that of the pristine HPC due to some residual THQ in the QS fraction. The oxygen content of HPC-MNS of $3.46 \mathrm{wt} \%$ was marginally higher than that of pristine HPC of $2.95 \mathrm{wt} \%$, while the oxygen content of HPC-QS decreased to $2.27 \mathrm{wt} \%$. Moreover, HPC-MNS consisted of $f_{a}$ of 0.66 and $M_{w}$ of $390 \mathrm{~m} / z$, while HPC-QS had $f_{a}$ of 0.63 , and $M_{w}$ of $269 \mathrm{~m} / z$. Both of their $f_{a}$ and $M_{w}$ were lower than those of original HPC. 
Table 1. Characteristics of the raw materials.

\begin{tabular}{cccccc}
\hline Raw Materials & Yield (\%) $^{\mathbf{a}}$ & $\mathbf{S P}\left({ }^{\circ} \mathbf{C}\right)$ & $\mathbf{O}(\mathbf{w t} \mathbf{\%})$ & $f_{a}{ }^{\mathbf{c}}$ & $\boldsymbol{M}_{\boldsymbol{w}}{ }^{\mathbf{d}}$ \\
\hline HPC & 45 & - & 2.95 & 0.77 & 472 \\
HPC-MNS & 23 & 170 & 3.46 & 0.66 & 390 \\
HPC-QS & 48 & 90 & 2.27 & 0.63 & 269 \\
\hline
\end{tabular}

Yield ${ }^{\mathrm{a}}$ was calculated based on the coal; $\mathrm{O}^{\mathrm{b}}=100-\mathrm{C}-\mathrm{H}-\mathrm{N} ; f_{a}{ }^{\mathrm{c}}$ : carbon aromaticity degree was calculated from ${ }^{13} \mathrm{C}-\mathrm{NMR}$ result; $M_{w}$ : average molecular weight was calculated from TOF-MS result.

The ${ }^{13} \mathrm{C}-\mathrm{NMR}$ spectra of HPC, HPC-MNS and HPC-QS are shown in Figure 1. The primary peak appears around 100 to $150 \mathrm{ppm}$ in all of the spectra, which is the aromatic carbon peak. Meanwhile there are two peaks deriving from the aliphatic carbon in the region around 10 to $50 \mathrm{ppm}$. A stronger peak is observed at $18 \mathrm{ppm}$, indicating that the HPC has amount of the aliphatic carbon in the form of methyl attached to an aromatic ring. A smaller peak appears at $38 \mathrm{ppm}$, deriving from either the saturated rings within polyaromatic sheets of HPC or from the ethylene bridges between two aromatic sheets. Usually, there are two modified outcomes of their carbon structures in the coal after hydrogenation. One is the hydrogen can saturate the aromatic rings and double or triple bond compounds to form naphthenic structure, thus increasing the intensity of peak in the region around 30 to $50 \mathrm{ppm}$ of the ${ }^{13} \mathrm{C}-\mathrm{NMR}$ spectra. Alternatively, the hydrogen can break the ethylene or heteroatoms linking bridges between the aromatic units, showing that the intensity of peak in the region around 30 to $50 \mathrm{ppm}$ of the ${ }^{13} \mathrm{C}-\mathrm{NMR}$ spectra decreases [18]. From our results, in comparison to HPC or HPC-MNS, the ${ }^{13} \mathrm{C}-\mathrm{NMR}$ spectra of HPC-QS shows that there is an obvious increased intensity of peak in the region around 30 to $50 \mathrm{ppm}$. For this reason, it was certified that most of the hydrogen was incorporated to aromatic rings and double or triple bond compounds, increasing the naphthenic structure after hydrogenation treatment of HPC with THQ. Meanwhile, this revealed that the hydrogen has successfully facilitated breakage of cross-linking bridges between the aromatic units in the HPC structure, providing the resultant molecular structure with short alkyl chains and light matters in the HPC-QS precursor. This finding also related to the SP and spinnability behavior of resulting HPC fractions. The molecular weight distribution of the two precursors produced HPC-MNS and HPC-QS, as well as of the original HPC, are shown in Figure 2. HPC has broad molecular weight distribution of 200 to $900 \mathrm{~m} / \mathrm{z}$, while the MNS fraction and QS fraction of HPC have narrow molecular weight distribution of 200 to $750 \mathrm{~m} / \mathrm{z}$, which indicated that the heavy molecular components of pristine HPC were obviously cut off by 1-MN solvent extraction or hydrogenation with THQ. Furthermore, TGA curves showed that the thermal stability properties of HPC, HPC-MNS and HPC-QS as show in Figure 3. The weight of original HPC was sustained decreased from 100 to $800^{\circ} \mathrm{C}$, and the decomposition temperature of HPC-MNS was about $300{ }^{\circ} \mathrm{C}$. However, the HPC-QS was decomposed only between 200 and $500{ }^{\circ} \mathrm{C}$, which indicated the HPC after hydrogenation treatment became more homogenous. Additionally, there is a broad peak between $10^{\circ}$ and $30^{\circ}$ in the XRD patterns of HPC, HPC-MNS and HPC-QS fractions (Figure 4). Peak in the $10^{\circ}$ to $20^{\circ}$ rang of XRD pattern is derived from the amorphous structure, while Peak in the $20^{\circ}$ to $30^{\circ}$ range is attributed to the stacked molecular structures [19]. Therefore, from the XRD results, HPC-QS contained more amorphous structures and a lower degree of molecular stacking than did HPC or HPC-MNS in accordance with the lower $f_{a}$ for HPC-QS. Clearly, these results indicate that significant differences exist in the molecular structures and compositions of HPC-MNS and HPC-QS, which would influence the utility of MNS and QS fractions of HPC for preparing spinnable pitches and the resulting CFs. 


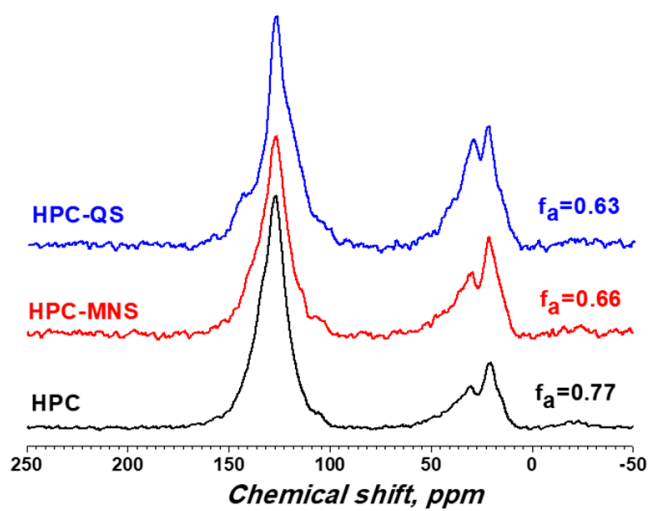

Figure 1. ${ }^{13} \mathrm{C}-\mathrm{NMR}$ spectra of HPC, HPC-MNS and HPC-QS.

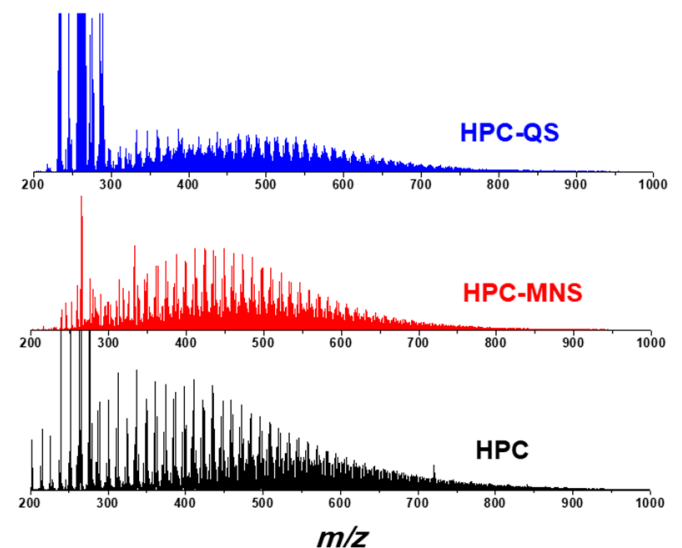

Figure 2. Molecular weight distribution of HPC, HPC-MNS and HPC-QS.

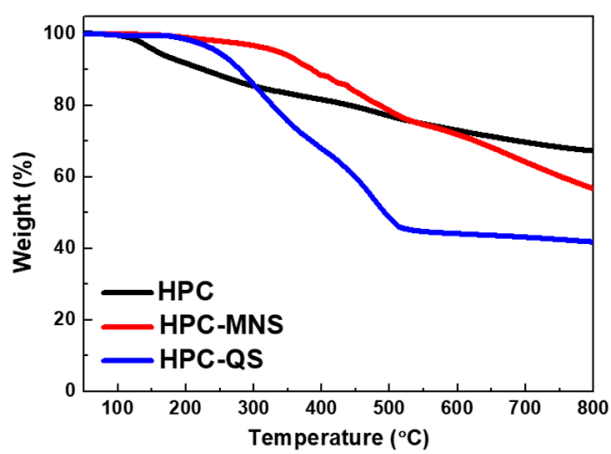

Figure 3. TGA curves of HPC, HPC-MNS and HPC-QS with a $100 \mathrm{~mL} / \mathrm{min}$ nitrogen flow rate.

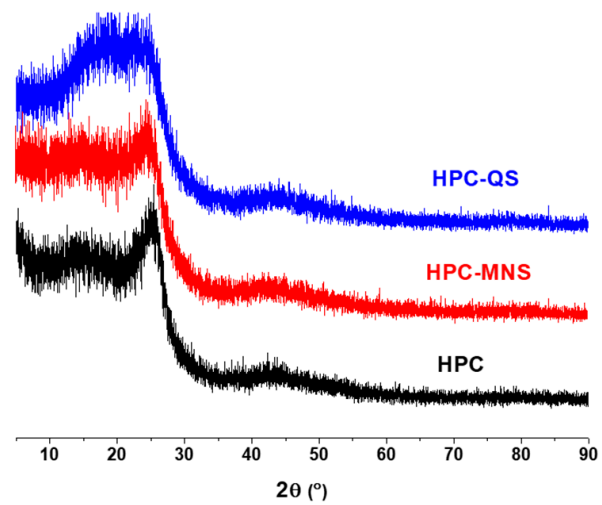

Figure 4. XRD patterns of HPC, HPC-MNS and HPC-QS. 


\subsection{Characterization of Prepared Pitches}

The properties of the resulting pitches with SPs of 210,240 and $260^{\circ} \mathrm{C}$ are summarized in Table 2. The SPs of HPC-MNS or HPC-QS derived pitches increased with concurrent decreases in their yield, which was due to the removal of low molecular weight compounds and slight polymerization of the precursors during the thin layer evaporation process. Moreover, HPC-QS derived pitch had a higher pitch yield than did HPC-MNS derived pitch at the same SP. Both of HPC-MNS and HPC-QS derived pitch had higher marginally oxygen content than the original precursor, respectively. In addition the oxygen content of obtained pitch slightly increased with increasing SP.

Table 2. Properties of the prepared pitches.

\begin{tabular}{lccccc}
\hline \multicolumn{1}{c}{ Pitches } & Yield (\%) & $\mathbf{O}(\mathbf{w t} \boldsymbol{\%})$ & $f_{\boldsymbol{a}}$ & $\boldsymbol{M}_{\boldsymbol{w}}$ & Number of Fiber Breakage * $^{*}$ \\
\hline HPC-MNS-SP210 & 21 & 2.98 & 0.68 & 494 & 0 \\
HPC-MNS-SP240 & 20 & 2.97 & 0.73 & 513 & 2 \\
HPC-MNS-SP260 & 18 & 3.01 & 0.74 & 578 & 5 \\
HPC-QS-SP210 & 35 & 2.38 & 0.67 & 469 & 0 \\
HPC-QS-SP240 & 31 & 2.44 & 0.70 & 489 & 0 \\
HPC-QS-SP260 & 29 & 2.54 & 0.74 & 498 & 0 \\
\hline
\end{tabular}

${ }^{*}$ During the $3 \mathrm{~min}$ spinning time at the rotation speed of winder of $600 \mathrm{~m} / \mathrm{min}$.

The resulting pitches were also analyzed by element analyses, ${ }^{13} \mathrm{C}-\mathrm{NMR}$ (Figure 5), and TOF-MS (Figure 6). ${ }^{13} \mathrm{C}-\mathrm{NMR}$ analyses indicated that the $f_{a}$ of obtained pitch increased with increasing SP due to the removal and polymerization of light matters (Table 2). Meanwhile, there is a gradually decreased intensity of peak in the region around 30 to $50 \mathrm{ppm}$ of the ${ }^{13} \mathrm{C}-\mathrm{NMR}$ spectra, which indicated that the cross-linking bridges between the aromatic units were further broken during the thin layer evaporation process. TOF-MS results showed that the molecular weight distributions of HPC-MNS and HPC-QS derived pitches were between 250 and $800 \mathrm{~m} / z$, indicating that low molecular weight compounds in the range of 200-500 $\mathrm{m} / z$ were successfully removed. Moreover, the $M_{w}$ of HPC-QS derived pitch was lower than that of HPC-MNS derived pitch at the same SP (Table 2), which suggested that the HPC-QS derived pitch had more homogenous than the HPC-MNS derived pitch. Additionally, Figure 7 shows XRD patterns of HPC-MNS and HPC-QS derived pitches. Whether HPC-MNS derived pitches or HPC-QS derived pitches, their peaks between $20^{\circ}$ and $30^{\circ}$ increased as increasing their SP, which was in agreement with their higher $f_{a}$ values with higher SP. Hence, these results revealed that pitches with higher SPs contain a higher degree of molecular structure stacking and less amorphous molecular structure.
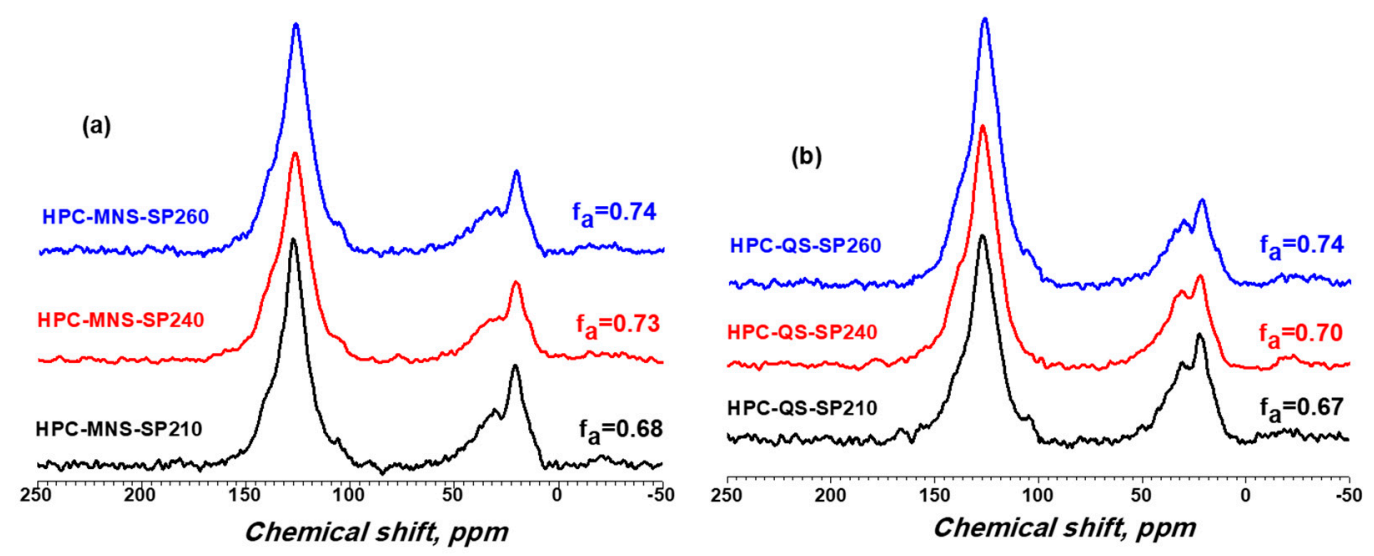

Figure 5. ${ }^{13} \mathrm{C}-\mathrm{NMR}$ spectra of HPC-MNS (a) and HPC-QS (b) derived pitches. 

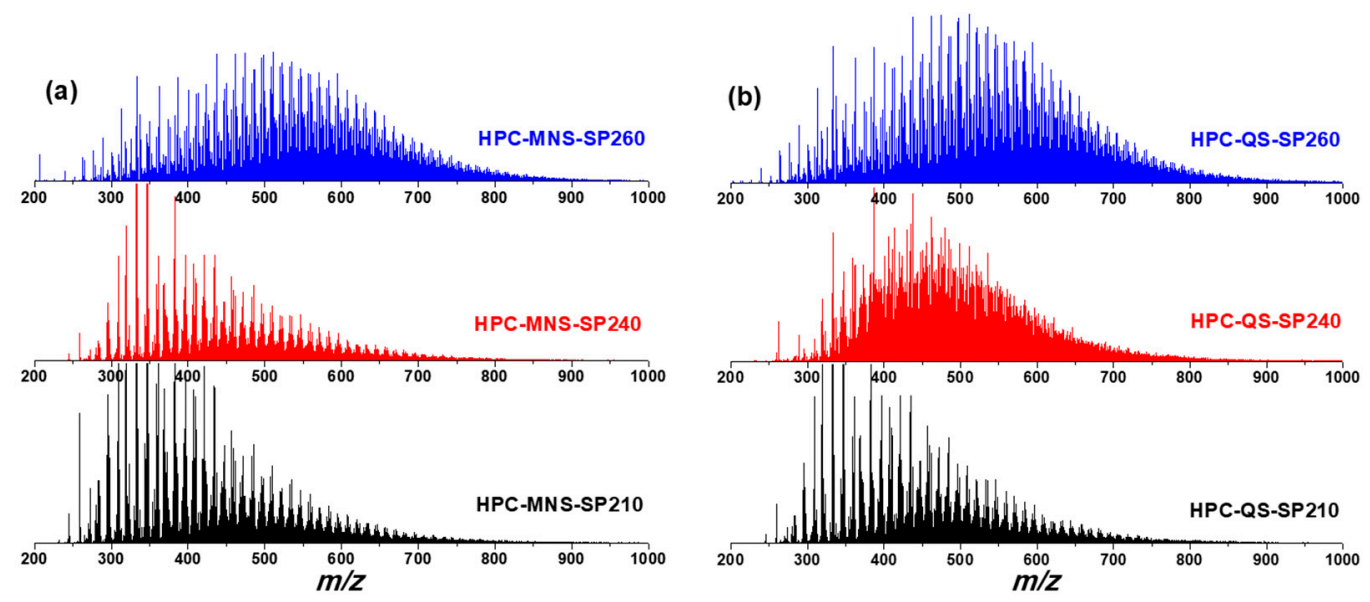

Figure 6. Molecular weight distribution of HPC-MNS (a) and HPC-QS (b) derived pitches.
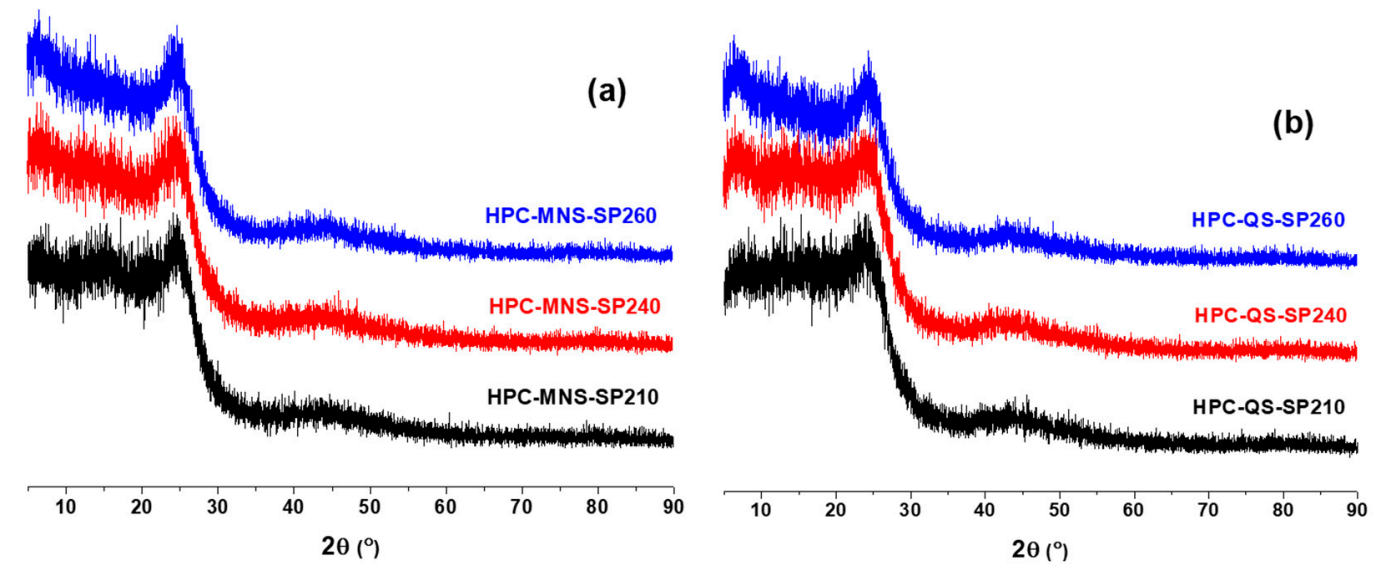

Figure 7. XRD patterns of HPC-MNS (a) and HPC-QS (b) derived pitches.

For the comparison of their spinnability, the HPC-QS derived pitches even with the SP of $260^{\circ} \mathrm{C}$ could be continuous to spin into pitch fibers without the fiber breakage during the more than $12 \mathrm{~min}$ spinning time. While the HPC-MNS derived pitches with the SP over $210^{\circ} \mathrm{C}$ arose many fiber breakage problems during the $3 \mathrm{~min}$ spinning time at the rotation speed of winder of $600 \mathrm{~m} / \mathrm{min}$ (Table 2), indicating bad spinnability. Thus, the spinning results of HPC-MNS and HPC-QS derived pitches indicated that the spinnability of HPC derived pitch could be improved by using QS fraction as a precursor.

Therefore, these analytical data suggested that the HPC-QS derived pitch showed higher pitch yield, higher SP, more homogenous and better spinnability than HPC-MNS derived pitch, which was due to lower oxygen content, and $f_{a}$ and $M_{w}$ of HPC-QS precursor than those of HPC-MNS precursor.

\subsection{Stabilization and Carbonization Process of Pitch Fibers}

Figure 8 shows the yield and oxygen contents of SFs and CFs prepared from HPC-MNS and HPC-QS derived pitch fibers. It is noticed that the stabilization yield and carbonization yield of HPC-MNS-derived fibers decreased with increasing SP of their pitches. In contrast, those of HPC-QS-derived fibers increased with increasing SP of their pitches. However, the oxygen contents of HPC-MNS-derived fibers and HPC-QS-derived fibers decreased with increasing SP of their pitches due to their higher aromaticity of pitch precursors. Moreover, whether stabilized fibers or carbonized fibers, the oxygen contents of HPC-QS-derived fibers was higher than those of HPC-MNS-derived fibers even the oxygen contents of HPC-QS-derived pitches was lower than those of HPC-MNS-derived pitches. 
It reveals that the HPC-QS-derived pitch fibers are easier to be oxidized than the HPC-MNS-derived pitch fibers due to the HPC-QS-derived pitches containing lower $f_{a}$ and $M_{w}$.

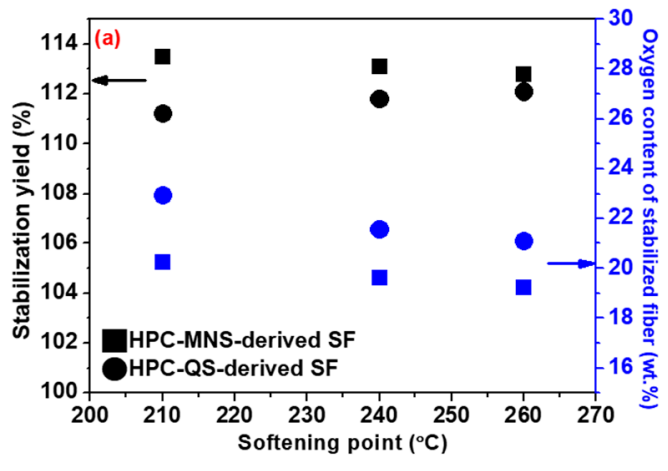

(a)

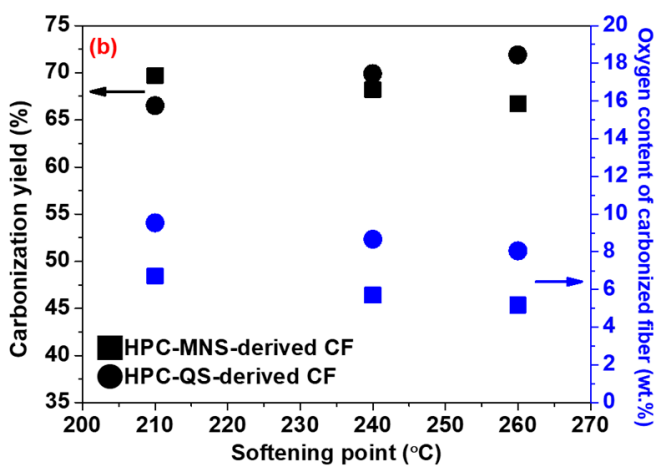

(b)

Figure 8. Yield and oxygen contents of (a) SFs and (b) CFs.

\subsection{Morphology and Mechnical Property of Obtained CFs}

The SEM of CFs obtained from the HPC-MNS-SP240 and HPC-QS-SP260 derived pitches as shown in Figure 9. The obtained CFs with a diameter of about $8 \mu \mathrm{m}$ showed a smooth surface morphology, and exhibited no fusing between the fibers, indicating that the stabilization and carbonization conditions had been appropriate. In addition, the cross section of the CFs prepared from HPC-MNS-SP240 derived pitch had some pores (Figure 9b), due to the less homogeneous melting state of this pitch with high SP of $240{ }^{\circ} \mathrm{C}$. However, the cross-section of the CFs prepared from HPC-QS-SP260 derived pitch showed a homogeneous and glass-like fracture surface (Figure 9d), indicating that the HPC derived CFs with good performance had been successfully prepared using the HPC-QS fraction as a precursor.
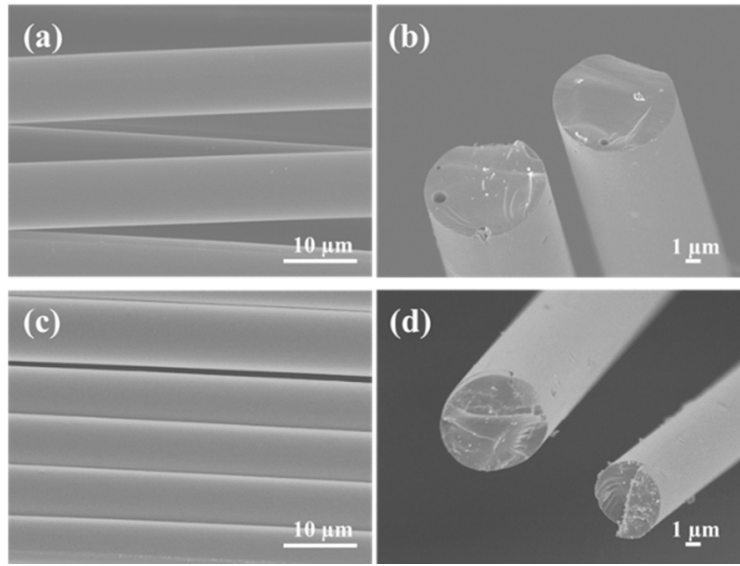

Figure 9. SEM of CFs obtained from (a,b) HPC-MNS-SP240; (c,d) HPC-QS-SP260 derived pitches ((a,c) are surface section of fibers, $(\mathbf{b}, \mathbf{d})$ are cross-section of fibers).

The tensile strengths of the CFs obtained from HPC-MNS and HPC-QS-derived pitches was shown in Figure 10. It is noticed that the tensile strengths of CFs increased as the higher SP of pitches due to the higher aromaticity of pitches with higher SP. Moreover, the CFs prepared from HPC-QS derived pitches had higher tensile strengths than the CFs prepared from HPC-MNS derived pitches. This was due to the HPC-QS fractions had been successfully modified by hydrogenation with THQ to decrease the oxygen content and adjust the molecular structure of HPC, the resulting HPC-QS 
derived pitch showed more homogeneous melted state and excellent spinnability even with higher SP. Therefore, the tensile strength of CFs obtained from HPC-QS-SP260 derived pitch was up to $1350 \mathrm{MPa}$, which was much higher than that of the commercial ones of 600-800 $\mathrm{MPa}$ [20].

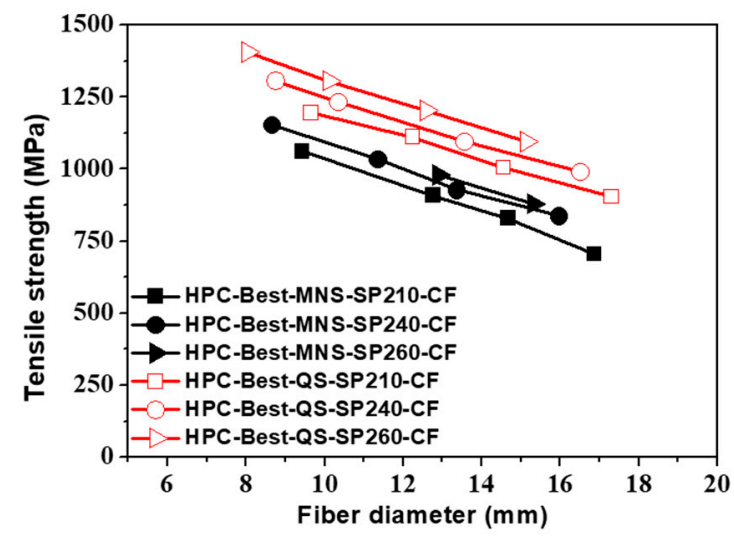

Figure 10. Tensile strengths of CFs obtained from HPC-MNS and HPC-QS derived pitches.

\section{Conclusions}

The SP of HPC-derived spinnable pitch with excellent spinnability increased to $260{ }^{\circ} \mathrm{C}$ using HPC-QS faction as a precursor and the tensile strength of resultant CFs was up to $1350 \mathrm{MPa}$ with a diameter around $8 \mu \mathrm{m}$ under the carbonization temperature of only $800{ }^{\circ} \mathrm{C}$ for $5 \mathrm{~min}$. Thus, this reported work revealed that the QS fraction of HPC was a valuable alternative for developing low-cost isotropic pitch based CFs with high tensile strength, and further suggested that it was feasible way to achieve the target of low-cost isotropic pitch based CFs with high performance production using HPC as an effective and low-price raw material. Moreover, the present results showed the oxygen contents, molecular structure of pitch precursor was very important effects on the spinning, stabilization and carbonization process. Thus, we are continuing to investigate the evaluation of oxygen functional groups of HPC-derived pitches and fibers in the current work.

Author Contributions: J.Y. and S.-H.Y. conceived and designed the experiments; J.Y., W.W., X.Z. and K.S. performed the experiments; J.Y., X.L. and S.-H.Y. analyzed the data; J.Y. and S.-H.Y. contributed reagents/materials/analysis tools; J.Y. wrote the paper.

Funding: This research was funded by the National Science Foundation for Young Scientists of China (Grant No. 51702094) and the Natural Science Foundation for Young Scientists of Hunan Province, China (Grant No. 2017JJ3014).

Acknowledgments: The authors appreciate the support of Kobe Steel, Ltd. for providing HPCs.

Conflicts of Interest: The authors declare no conflict of interest.

\section{References}

1. Baker, D.A.; Rials, T.G. Recent advances in low-cost carbon fiber manufacture from lignin. J. Appl. Polym. Sci. 2013, 130, 713-728. [CrossRef]

2. Ma, X.J.; Zhao, G.J. Preparation of carbon fibers from liquefied wood. Wood Sci. Technol. 2010, 44, 3-11.

3. Berrueco, C.; Álvarez, P.; Díez, N.; Granda, M.; Menéndez, R.; Blanco, C.; Santamaria, R.; Millan, M. Characterisation and feasibility as carbon fibre precursors of isotropic pitches derived from anthracene oil. Fuel 2012, 101, 9-15. [CrossRef]

4. Prauchner, M.J.; Pasa, V.M.; Otani, S.; Otani, C. Biopitch-based general purpose carbon fibers: Processing and properties. Carbon 2005, 43, 591-597. [CrossRef]

5. Ko, S.; Choi, J.E.; Lee, C.W.; Jeon, Y.P. Modified oxidative thermal treatment for the preparation of isotropic pitch towards cost-competitive carbon fiber. J. Ind. Eng. Chem. 2017, 54, 252-261. [CrossRef] 
6. Kim, B.J.; Eom, Y.H.; Kato, O.; Miyawaki, J.; Kim, B.C.; Mochida, I.; Yoon, S.H. Preparation of carbon fibers with excellent mechanical properties from isotropic pitches. Carbon 2014, 77, 747-755. [CrossRef]

7. Kim, B.J.; Eom, Y.; Kato, O.; Miyawaki, J.; Kim, B.C.; Mochida, I.; Yoon, S.H. Enhancing the tensile strength of isotropic pitch-based carbon fibers by improving the stabilization and carbonization properties of precursor pitch. Carbon 2016, 99, 649-657. [CrossRef]

8. Yang, J.; Nakabayashi, K.; Miyawaki, J.; Yoon, S.H. Preparation of isotropic pitch-based carbon fiber using Hyper-coal through co-carbonation with ethylene bottom oil. J. Ind. Eng. Chem. 2016, 34, 397-404. [CrossRef]

9. Yang, J.; Nakabayashi, K.; Miyawaki, J.; Yoon, S.H. Preparation of pitch based carbon fibers using Hyper-coal as a raw material. Carbon 2016, 106, 28-36. [CrossRef]

10. Hamada, T.; Nishida, T.; Sajiki, Y.; Matsumoto, M.; Endo, M. Structures and physical properties of carbon fibers from coal tar mesophase pitch. J. Mater. Res. 1987, 2, 850-857. [CrossRef]

11. Hatano, H.; Suginobe, H. Improvement and control of the quality of binder pitch for graphite electrodes. Fuel 1989, 68, 1503-1506. [CrossRef]

12. Daguerre, E.; Guillot, A.; Stoeckli, F. Activated carbons prepared from thermally and chemically treated petroleum and coal tar pitches. Carbon 2001, 8, 1279-1285. [CrossRef]

13. Brage, C.; Yu, Q.; Chen, G.; Sjöström, K. Tar evolution profiles obtained from gasification of biomass and coal. Biomass Bioenerg. 2000, 18, 87-91. [CrossRef]

14. Okuyama, N.; Komatsu, N.; Shigehisa, T.; Kaneko, T.; Tsuruya, S. Hyper-coal process to produce the ash-free coal. Fuel Process. Technol. 2004, 85, 947-967. [CrossRef]

15. Wang, P.; Jin, L.; Liu, J. Analysis of coal tar derived from pyrolysis at different atmospheres. Fuel 2013, 104, 14-21. [CrossRef]

16. Takanohashi, T.; Shishido, T.; Kawashima, H.; Saito, I. Characterisation of Hyper-Coals from coals of various ranks. Fuel 2008, 87, 592-598. [CrossRef]

17. Mishra, A.; Saha, M.; Bhatia, G.; Aggarwal, R.K.; Raman, V.; Yadav, H.S. A comparative study on the development of pitch precursor for general-purpose carbon fibers. J. Mater. Process. Technol. 2005, 168, 316-320. [CrossRef]

18. Andrésen, J.M.; Zhang, Y.; Burgess, C.E.; Schobert, H.H. Synthesis of pitch materials from hydrogenation of anthracite. Fuel Process. Technol. 2004, 85, 1361-1372. [CrossRef]

19. Andersen, S.I.; Jensen, J.O.; Speight, J.G. X-ray diffraction of subfractions of petroleum asphaltenes. Energ. Fuel 2005, 19, 2371-2377. [CrossRef]

20. Liu, Y.; Kumar, S. Recent progress in fabrication, structure, and properties of carbon fibers. Polym. Rev. 2012, 42, 21-32. [CrossRef]

(C) 2018 by the authors. Licensee MDPI, Basel, Switzerland. This article is an open access article distributed under the terms and conditions of the Creative Commons Attribution (CC BY) license (http://creativecommons.org/licenses/by/4.0/). 